\title{
On monotone multivalued functions: generating and estimating Dedekind numbers
}

\author{
Luís Henrique de Santana, $\quad \underline{\text { André Leite }}$ \\ Departamento de Estatística, CCEN, UFPE \\ 50740-540, Recife, PE \\ E-mail: desantanalh@gmail.com, leite.andre@gmail.com
}

\begin{abstract}
Monotone multivalued functions have been widely employed in discrete mathematics, multivalued logics and reliability theory, in special its particular case of monotone boolean functions. However, our interest in them came from the kind of monotone cellular automata studied in $[1,2,3,4,7,8,9,10,11,12,13]$, where monotone multivalued functions play a fundamental role. At first, we present an algorithm that generates random examples of monotone multivalued functions. Our second algorithm provide an estimative for the number of monotone multivalued functions, which shall be called here Dedekind number. Our first algorithm provided examples that were used for testing routines that compute Galperin's rates [9]. Moreover, it will facilitate the inductive reasoning concerning monotone cellular automata.
\end{abstract}

Keywords: monotone boolean function, monotone multivalued function, Dedekind number

\section{Introduction}

Let $m$ and $k$ be natural numbers. The set $M_{m}=\{0,1, \ldots, m\}$ is called the set of states. Let $v=\left(v_{1}, \ldots, v_{k}\right)$ and $u=\left(u_{1}, \ldots, u_{k}\right)$ be elements of $M_{m}^{k}$. We denote

$$
v \prec u \Leftrightarrow v_{1} \leq u_{1}, \ldots, v_{k} \leq u_{k} .
$$

Notice that $\prec$ defines a partial order.

Any function $f: M_{m}^{k} \rightarrow M_{m}$ is called a multivalued. A multivalued function $f: M_{m}^{k} \rightarrow M_{m}$ is called monotone if

$$
\forall v, u \in M_{m}^{k}: v \prec u \Rightarrow f(v) \leq f(u) .
$$

Notice that a multivalued function $f: M_{m}^{k} \rightarrow M_{m}$ is monotone if and only if it satisfies the following set of integer inequalities:

$$
\begin{aligned}
& 0 \leq f\left(a_{1}, a_{2}, \ldots, a_{k}\right) \leq m \text { for all } a_{1}, a_{2}, \ldots, a_{k} \in M_{m} \\
& 0 \leq f\left(a_{1}+1, a_{2}, \ldots, a_{k}\right)-f\left(a_{1}, a_{2}, \ldots, a_{k}\right) \text { for all } a_{2}, a_{3}, \ldots, a_{k} \in M_{m}, a_{1} \in M_{m-1} \\
& 0 \leq f\left(a_{1}, a_{2}+1, \ldots, a_{k}\right)-f\left(a_{1}, a_{2}, \ldots, a_{k}\right) \text { for all } a_{1}, a_{3}, \ldots, a_{k} \in M_{m}, a_{2} \in M_{m-1} \\
& 0 \leq f\left(a_{1}, a_{2}, \ldots, a_{k}+1\right)-f\left(a_{1}, a_{2}, \ldots, a_{k}\right) \text { for all } a_{2}, \ldots, a_{k-1} \in M_{m}, a_{k} \in M_{m-1}
\end{aligned}
$$

Sometimes it is also assumed that

$$
f(a, \ldots, a)=a \text { for all } a \in M_{m} .
$$

Monotone multivalued functions have been widely employed in discrete mathematics, multivalued logics and reliability theory, in special its particular case of monotone boolean functions. However, our interest in them came from the kind of cellular automata studied in 
$[1,2,4,7,8,9,10,11,12,13]$, where monotone multivalued functions satisfying assumption (3) play a fundamental role.

Counting the number of monotone functions from $M_{1}^{k}$ to $M_{1}$ is a classical problem. This number is known as Dedekind number. Here the number of monotone multivalued functions from $M_{m}^{k}$ to $M_{m}$ shall be also called Dedekind number.

Our questions are:

1. Given $k$ and $m$, how can we can generate a random example of monotone multivalued function?

2. Given multivalued function, how can we estimate Dedekind numbers for multivalued monotone functions?

\section{Generating}

The map $d: M_{m}^{k} \times M_{m}^{k} \rightarrow \mathbb{R}_{+}$given by

$$
d\left(v, v^{\prime}\right)=\sum_{i=1}^{k}\left|v_{i}-v_{i}^{\prime}\right|
$$

is a metric. The simple oriented graph $H_{m, k}$ where the set of vertices is $M_{m}^{k}$ and the set of edges is

$$
E_{m, k}=\left\{\left(v, v^{\prime}\right) \in M_{m}^{k} \times M_{m}^{k}: d\left(v, v^{\prime}\right)=1, d(v, 0)<d\left(v^{\prime}, 0\right)\right\}
$$

is called Hasse diagram of $M_{m}^{k}$. The adjancency matrix of graph $H_{m, k}$ is denoted by $\mathcal{A}_{m, k}$.

A partial order $\prec^{*}$ on the set $M_{m}^{k}$ in an extension of another partial order $\prec$ on the set $M_{m}^{k}$ if

$$
\forall v, u \in M_{m}^{k}: v \prec u \Rightarrow v \prec * u .
$$

A linear extension is an extension that is also a total order.

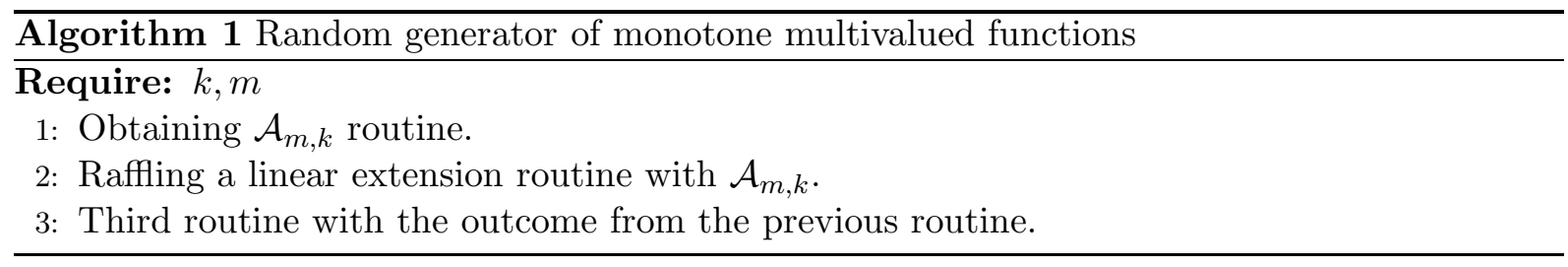

The Raffling a linear extension routine was obtained by modifying the topological ordering algorithm [5] and using some ideas presented in [6]. This routine requires the partial order $\prec$ on the set $M_{m}^{k}$ represented by the adjacency matrix $\mathcal{A}_{m, k}$ and returns a random example of linear extension $\prec^{*}$ of $\prec$ on the set $M_{m}^{k}$.

Let us present the idea of the Third routine. After the second routine we have

$$
(0,0, \ldots, 0)=p_{1} \prec^{*} p_{2} \prec^{*} \ldots \prec^{*} p_{(m+1)^{k}}=(m, m, \ldots, m),
$$

where $p_{j}$ denotes the $j$-th element according the total order and $j \in\left\{1,2, \ldots,(m+1)^{k}\right\}$. Suppose that the outcome of an uniform discrete distribution in the integer interval $\left[1,(m+1)^{k}\right]$ is $j_{1}$. Then $f\left(p_{j}\right)=0$ for all $j<j_{1}$ and $f\left(p_{j_{1}}\right)=1$. If $j_{1}=(m+1)^{k}$, then it is over. Otherwise, suppose that the outcome of an uniform discrete distribution in the interval $\left[j_{1}+1,(m+1)^{k}\right]$ is $j_{2}$. Then $f\left(p_{j}\right)=1$ for all $j_{1}<j<j_{2}$ and $f\left(p_{j_{2}}\right)=2$. If $j_{2}=(m+1)^{k}$, then it is over. Otherwise, suppose that the outcome of an uniform discrete distribution in the integer interval $\left[j_{2}+1,(m+1)^{k}\right]$ is $j_{3}$ and so on.

Theorem 1 Given $k$ and $m$, the outcome of Algorithm 1 is a random variable whose sample space is the set of all monotone multivalued functions from $M_{m}^{k}$ to $M_{m}$. 
We have implemented Algorithm 1 and a modified version of Algorithm 1 whose outcome is a random variable where its sample space is the set of all monotone multivalued functions from $M_{m}^{k}$ to $M_{m}$ satisfying (3).

\section{Estimating Dedekind number}

Consider the set $\mathcal{F}_{m, k}$ of all multivalued functions from $M_{m}^{k}$ to $M_{m}$. We know that $\mathcal{F}_{m, k}$ has $(m+1)^{(m+1)^{k}}$ elements. However, how may among these elements are monotone?

Let $n$ be a natural number. Algorithm 2 estimates the proportion of monotone multivalued functions in the set $\mathcal{F}_{m, k}$. The system of integer inequalities (2) can be used for testing

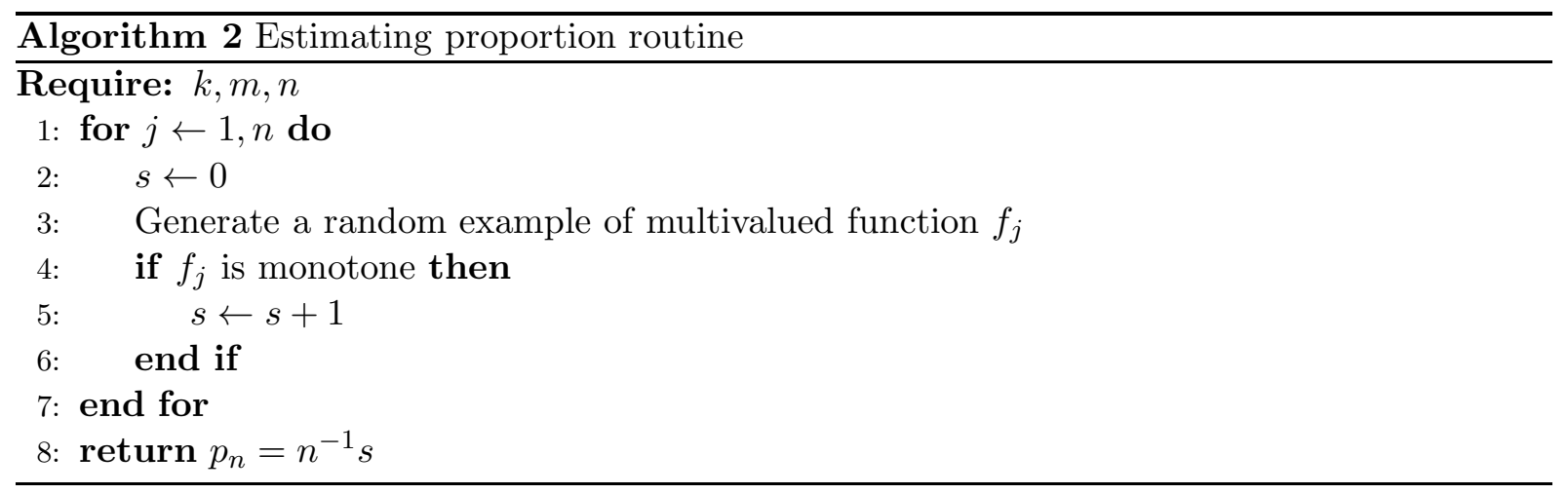

monotonicity of multivalued function $f_{i}$ in Algorithm 2.

Lemma 1 The sequence $\left\{p_{n}(m+1)^{(m+1)^{k}}\right\}_{n \in \mathbb{N}}$ converges in probability to the Dedekind number.

\section{Conclusions}

Algorithm 2 is not implemented yet. The modified version of Algorithm 1 provided examples that were used for testing routines that compute Galperin's rates [9]. Moreover, Algorithm 1 will facilitate the inductive reasoning concerning monotone cellular automata.

\section{Acknowledgments}

L. H. de Santana thanks CNPq-Brazil for the financial support.

\section{Referências}

[1] R. Fernándes and A. Toom, Non-gibssianness of the invariant measures of non-reversible cellular automata with totally asymmetric noise, Astérisque, 287 (2003) 71-87.

[2] G. A. Galperin, One-dimensional automaton networks with monotonic local interaction, Problems of Information Transmission, 12 (1975) 74-87.

[3] G. A. Galperin, One-dimensional local monotone operators with memory, Soviet Math. Dokl. 17 (1976), pp. 688-692.

[4] G. A. Galperin, Rates of interaction in one-dimensional networks, Problems of Information Transmission, 13 (1977) 73-81.

[5] A. B. Kahn, Topological sorting of large networks, Communications of the ACM, 5 (1962) 558-562. 
[6] A. Leite, "A edução da função utilidade multiatributo", Ph.D. Thesis, CTG-UFPE, 2008.

[7] M. Lima de Menezes and A. Toom, A non-linear eroder in presence of one-sided noise, Brazilian Journal of Probability and Statistics, 20 (2006) 1-12.

[8] L. H. de Santana, "Velocities a la Galperin in continuous spaces", Ph.D. Thesis, DMatUFPE, 2012.

[9] L. H. de Santana, A. Leite and A. Toom, Computing directional Galperin's rates, (2013) Submitted to CMAC-SUL 2014.

[10] L. H. de Santana, A. Leite and A. Toom, Implementing the Toom's erodicity criteria via directional Galperin rates, (2013) In Preparation.

[11] L. H. de Santana, A. D. Ramos and A. Toom, Eroders on a plane with three states at a point, (2013) In Preparation.

[12] R. N. de Souza Pereira, "Ergodicidade de um eroder unidimensional com ruído aleatório", Master Thesis, DE-UFPE, 2005.

[13] A. Toom, Monotonic binary cellular automata, Problems of Information Transmission, 12 (1976) 33-37. 